crystals, through the incorporation of nonlinear optical nanoparticles. Other possibilities mentioned by the researchers include holograms with magnetic nanocrystals for magneto-optic applications and the incorporation of inorganic nanocrystals for multifunctional lightemitting devices.

STEVEN TROHALAKI

\section{Sequential Synthesis of Colloidal Type-II Core/Shell CdTe/CdSe Semiconductor Nanocrystals Demonstrated}

Semiconductor nanocrystals have been the subject of recent scientific and technological interest due to their promising potential applications, including photovoltaics and bio-imaging. In heterostructured nanocrystals, a higher-bandgap shell material can be grown onto a core material with a lower bandgap. Type II core-shell nanocrystals are materials engineered by their band offset $\left(\Delta E_{\mathrm{v}}\right)$, and in these structures, the band offsets are such that the energies of the conduction and valence bands of the shell are either both higher or both lower than those of the core. These materials can spatially separate and confine photo-generated holes and electrons. A common-cation system, CdTe (core)/CdSe (shell) nanocrystals are type II materials, with the majority of photogenerated holes confined in the CdTe core and the electrons in the CdSe shell. Recently, K. Yu and co-workers from the National Research Council of Canada have developed an efficient approach to the synthesis of high-quality CdTe/CdSe nanocrystals. For the CdSe shell, by control of various factors, the technique avoids the formation of a thicker CdSe nanocrystal layer resulting in a thinner shell, leading to a higher photolumines-

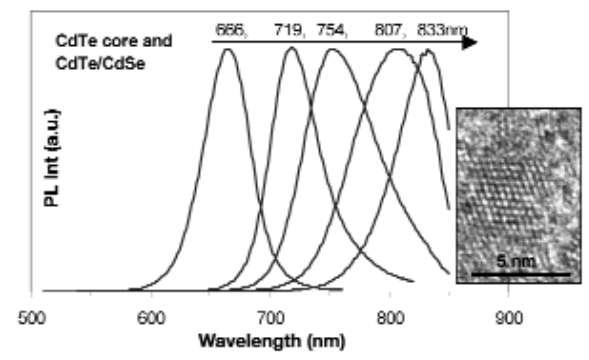

Figure 1. Photoemission spectra of $\mathrm{CdTe}$ (core)/ CdSe(shell) nanocrystals showing that the thicker the CoSe shell is, the more the emission redshifts. The inset is a transmission electron micrograph of one core-shell nanocrystal in an ensemble exhibiting $833 \mathrm{~nm}$ emission. cence efficiency. The researchers report their results in the March issue of Small.

Colloidal type II CdTe/CdSe nanocrystals were synthesized by the sequential addition of a tri-n-octylphosphine telluride (TOPTe)/tri- $n$-octylphosphine (TOP) solution and several shell-precursor solutions to a cadmium oxide (CdO)/TOP solution. The shell-precursor solutions consist of $\mathrm{CdO}$ and tri- $n$-octylphosphine selenide (TOPSe) in TOP. This synthetic approach is simple and does not involve either the addition of any acids, amines, or traditional tri-n-octylphosphine oxide (TOPO), or precipitation of core CdTe nanocrystals.

During the synthesis of the type-II $\mathrm{CdTe} / \mathrm{CdSe}$ nanocrystals, the researchers monitored the temporal evolution of the optical properties during the growth of the CdSe shell, as shown in the figure. The emission redshifts with increasing CdSe shell thickness.

This synthetic approach, similar to that of sequential anionic polymerization for well-defined block copolymers, is based on the knowledge gained during the search for synthetic routes for providing slow growth rates for high-quality CdSe and CdTe nanocrystals in large-scale production. It has been acknowledged that a slower particle growth rate results in less surface roughness, fewer surface defects, and higher photoluminescent efficiency. This synthetic approach is particularly well suited for realizing engineering materials with bandgaps in the nearinfrared and infrared spectral ranges.

GOPAL RAO

\section{Far-Field Technique for Visualization of Broadband Surface Plasmons Developed}

Surface plasmons (SPs) are traveling waves that propagate along the interface of a metal and an insulator. Commercially available sensors for environmental and biological applications have been fabricated by tailoring the interaction between impinging light and SPs. Recently, SPs have been explored as a means of realizing metallic photonic circuits. Imaging the SP intensity distribution is essential to characterizing SP-based devices. Traditionally, this imaging has been accomplished by near-field and fluorescence techniques. As reported in the April 15 issue of Optics Letters (p. 884), A. Bouhelier and G.P. Wiederrecht of the Chemistry Division and Center for Nanoscale Materials at Argonne National Laboratory have developed a far-field method for exciting and observing the SP intensity distribution. The SP intensity distribution was imaged by detecting leakage radiation, which are two of the four electromagnetic modes that are solutions to the dispersion relations.

The researchers deposited silver films onto glass substrates using thermal evaporation. To avoid total internal reflection of the radiative modes, an indexmatched, oil-immersed objective (numerical aperture of 1.4) was kept in contact with the glass substrate. The objective was part of an optical microscope, which was focused on the metal-glass interface. White light was used to excite a broadband surface plasmon continuum at the interface. This is possible because the objective introduced a distribution of wave vectors, through focusing, that fulfill the dispersion relation of the plasmon over the entire visible spectral region. The surface plasmon leakage radiation was recorded by a CCD camera that was placed in the image plane. A regeneratively amplified Ti:sapphire laser system was used to produce the incident whitelight continuum radiation, which was polarized by a multi-wavelength wave plate and a Glan-Thompson polarizer.

Propagating SPs were visualized by recording the real-space distribution of leakage radiation emitted by SP waves at the dielectric-metal interfaces. The images reveal a spatial variation in the spectral components of the SPs, which the researchers have termed "rainbow jets," that extend a significant distance along the direction of propagation of the SPs away from the incident continuum radiation spot. According to Bouhelier and Wiederrecht, the observation of surface plasmon leakage radiation gives a direct measurement of the SP propagation length and the damping mechanisms for each wavelength. The study of surface plasmons and light-SP interactions opens possibilities for photonics because they allow the concentration and propagation of light below the usual resolution limit, which may enable the realization of wavelength-sensitive optical devices.

JEREMIAH T. ABIADE

\section{Combination of Raman Scattering Techniques Achieve Single-Molecule Detection of Biomolecules}

As molecular biology advances, the detection of low quantities of biomolecules is proving critical for some applications, said researchers T.-W. Koo, S. Chan, and A.A. Berlin of the Precision Biology group at Intel Corporation in their article published in the May 1 issue of Optics Letters (p. 1024).

Surface-enhanced Raman scattering (SERS), which has been demonstrated as a single-molecule detection method, benefits from signal increases on the order of $10^{3}-10^{15}$ primarily because of the electro- 
magnetic field enhancements associated with surface plasmon resonances over the non-enhanced Raman scattering methods. However, SERS requires the target molecule to adsorb onto a metal surface to benefit from surface plasmon resonance. Consequently, it has been difficult to detect biological molecules that have a low affinity for metal surfaces, and only a few types of molecules have been detected by SERS with single-molecule sensitivity to date. The researchers said that huge signal gain can be obtained by combining SERS and coherent anti-Stokes Raman scattering (CARS) as is done in surfaceenhanced coherent anti-Stokes Raman scattering (SECARS). The researchers further improved the sensitivity of SECARS over the earlier reported work, and achieved single-molecule detection of some biomolecules not detected earlier using this method.

The researchers said that SECARS background originating from metal and water has limited the sensitivity of previous works. The research group used several methods, such as a polarizationsensitive CARS setup, minimized probe volume, and the use of aggregated colloidal silver nanoparticles instead of planar substrates, to reduce the background. The SECARS signals of biological molecules were increased by the addition of lithium chloride salts, which were found to be optimal for generating strong surface enhancements.

The researchers then demonstrated the power of SECARS by detecting two DNA nucleotides, deoxyadenosine monophosphate (dAMP) and deoxyguanosine monophosphate (dGMP), and a peptide-angiotensin-I peptide. Although dAMP has been detected at the single-molecule level by SERS, the SECARS signal was approximately 1000 times stronger than the SERS signal for dAMP at single-molecule concentrations. The SECARS signal of dGMP was at least 100 times stronger than the SERS signal of dGMP. The researchers believe that this is the first reported detection of dGMP at single-molecule concentrations.

The detection of the peptide shows that the SECARS technique can be applied to biological molecules other than nucleotides. According to the research group, the ultrasensitive detection of nonlabeled peptides and proteins has potential applications in pathogen detection, disease monitoring, and drug discovery.

VIVEK RANJAN

\section{Cell Membranes Integrated into CNT Devices}

Integrating biological systems and processes with nanofabricated structures is a critical challenge for nanotechnology. In the May issue of Nano Letters, J.-C. Gabriel, G. Grüner, and their co-workers reported on their work, performed at Nanomix Inc., on the integration of the cell membrane of Halobacterium salinarum with carbon nanotube (CNT) network transistors, wherein both the biological and nanoelectronic structures preserve their functionality and are able to electronically interact with each other.

The researchers fabricated nanotube network field-effect transistors, where the semiconducting channel is formed by a network of predominantly semiconducting nanotubes. A monolayer of the purple membrane from Halobacterium salinarum was then deposited on the exposed semiconductor channel. Evidence for the integration of the membrane with the device comes from changes in the device characteristics of the transistor.

The purple membrane contains bacteriorhodopsin, which has a permanent electric dipole moment pointing from the extracellular side of the membrane toward the cytoplasmic side. The researchers prepared three sets of devices. The first had the membrane dipole randomly oriented on the nanotubes, while the second and third sets had the cytoplasmic or extracellular side of the membrane facing the nanotubes. Prior to integration of the membrane, the nanotube devices by themselves worked as $p$-type transistors with good conduction at negative gate voltages and no conduction at positive gate voltages. The voltage at which the device turns on is known as the threshold voltage and is different depending on the sweep direction of the gate voltage (i.e., it is hysteretic). The hysteresis is known to be produced by water adsorbed on the substrate.

For the mixed-orientation nanobioelectronic device, deposition of the cell membrane resulted in a narrower hysteresis compared with the hysteresis of the nanotube devices alone. The researchers attributed this effect to desorption of water from the nanotube surface as the membrane displaced water. Also, the turn on for this device was less sharp, indicating a change in carrier mobility for the nanotube networks arising from random scattering potentials due to the electric dipoles of rhodopsin. The oriented membrane devices also showed a loss of hysteresis and a shift of the threshold voltage in different directions for the two different orientations of the membrane. Since the threshold voltage is correlated to the position of the Fermi level in nanotubes, the shift in the threshold voltage is indicative of a charge induced in the nanotube by the electric dipole of the membrane. The magnitudes of the shifts are different for the two orientations, implying asymmetry in the charge distribution of rhodopsin, with the dipole being closer to the cytoplasmic side.

Grüner, who is a professor at UCLA and advisor to the project, said that this work "opens up avenues for what could be called 'cellectronics,' the electronic detection and also modification of biological processes at the cellular level."

SARBAJIT BANERJEE

\section{Continuous-Wave $1.94 \mu \mathrm{m}$ Laser Based on $\mathrm{Tm}_{\mathrm{BaY}} \mathrm{B}_{2} \mathrm{~F}_{8}$ Lases from $1849 \mathrm{~nm}$ to $2059 \mathrm{~nm}$}

Efficient, widely tunable solid-state laser sources in the near-infrared region around $2 \mu \mathrm{m}$ have potential applications in remote sensing and gas detection, high-resolution spectroscopy, frequency metrology, and medicine. Thulium doping of crystals can be used in this wavelength region because of the ${ }^{3} \mathrm{~F}_{4}-{ }^{3} \mathrm{H}_{6}$ optical transition of $\mathrm{Tm}^{3+}$ ions that in most host media yields a very broad emission with a maximum at wavelengths of 1.8-1.9 $\mu \mathrm{m}$. In addition, $\mathrm{Tm}^{3+}$ has the advantage that it can be directly diodepumped at $\sim 785 \mathrm{~nm}$, and shows a quantum efficiency close to 2 (i.e., each pump quantum absorbed yields two Tm ions in the upper laser level).

In the April 15 issue of Optics Letters (p. 854), G. Galzerano and P. Laporta of Politecnico di Milano, in a joint collaboration with $\mathrm{M}$. Tonelli and colleagues from the Università di Pisa, Italy, described the growth, spectroscopic characterization, and laser action of a diode-pumped laser based on a Tm-doped fluoride crystal $\left(\mathrm{Tm}: \mathrm{BaY}_{2} \mathrm{~F}_{8}\right)$. They reported a roomtemperature, diode-pumped laser oscillator widely tunable over a $210 \mathrm{~nm}$ interval, from $1849 \mathrm{~nm}$ to $2059 \mathrm{~nm}$, with a maximum continuous-wave (cw) output power of $\sim 150 \mathrm{~mW}$ at $1920 \mathrm{~nm}$ by use of a $0.5 \%$ output coupler transmission.

The researchers said that fluoride crystals, despite thermomechanical properties that are usually poorer than those of most oxides, present some advantages in terms of laser performance. These include low phonon energy, longer fluorescence lifetime, lower upconversion losses, reduced thermal lensing, and extremely low-beam depolarization under strong pumping. The crystal was grown in a homemade Czochralski furnace, starting with $\mathrm{BaY}_{2} \mathrm{~F}_{8}$ powder and adding $\mathrm{BaF}_{2}$ and $\mathrm{TmF}_{2}$ powders. The best laser results were obtained for a Tm doping level of $12 \%$. Crystallinity and crystallographic orientation were determined from x-ray 